\title{
CASE OF A PATIENT
}

IN WHOM A

\section{FOREIGN BODY HAD BEEN INTRODUCED INTO} THE PELVIS THROUGH THE VAGINA,

\author{
AND \\ A DESCRIPTION OF A SUCCESSFUL OPERATION \\ FOR ITS REMOVAL.
}

\author{
BY JOHN C. W. LEVER, M.D., \\ PHYSICIAN-ACCOUCHEUR TO GUY'S HOSPITAL. \\ AND \\ JOHN HILTON, F.R.S., \\ ASSISTANT-SURGEON TO GUY'S HOSPITAL.
}

Received April 27th-Read June 13th, 1848.

$\mathrm{E}-\mathrm{P}-$ - 34 years of age, a woman of tall stature, fair complexion, and rather delicate appearance, was admitted into Petersham Ward, Guy's Hospital, under the care of Dr. Lever, Dec. 31, 1847.

Her general health is pretty good. She has had two attacks of acute rheumatism, one when 16 years of age, and another six years ago. Her relatives are stated to be consumptive, and her husband died of phthisis.

History.-On the 6th of June 1847, whilst in the act of applying some ointment on the end of a bone netting-mesh to allay a local irritation in the vagina, she was disturbed by a person entering the room, and suddenly sat down. This caused her great pain, and a small quantity of blood escaped 
from the vagina, the mesh being forced quite out of sight, so that only the extremity of it could be felt; and in consequence of her repeated fruitless attempts to remove it, was at length forced so far into the pelvis that she could not feel it. Immediately after the accident she discovered that she could not bend her body or sit down, being obliged to keep herself either in the erect or horizontal position.

She has ever since experienced severe pain in the right side of the pelvis, extending down the extremity of the same side : the thigh and leg often feel cold and benumbed. The pain is at times so acute as to make her afraid to attempt any movement, and it is always increased by any sudden jerk or motion, as treading on a stone, \&c.

During the summer months she was able to walk a short distance, and to go up and down stairs without any great inconvenience : but in November her health became impaired; and the pain was so severe as to confine her to bed, and to require large doses of opium before any relief was obtained.

Before admission into the hospital, she was seen by $\mathrm{Mr}$. Callaway, who requested Dr. Lever to examine the uterus: this was performed by the finger, and by the uterine sound, but he could detect nothing in its cavity. Subsequently the case was treated, by another physician who was consulted, as one of severe sciatica.

Symptoms on admission.- She appears much exhausted and emaciated from the protracted suffering she has endured; she complains of severe pains extending from the posterior part of the thigh and leg to the heel ; this pain is at times much more intense than at others, and during the paroxysms she obtains some relief by extending the limb as much as possible. The pain appears to follow pretty exactly the course of the great ischiatic nerve, over the situation of which, as it emerges from the pelvis, there is much tenderness on pressure. There is also some amount of tenderness in the right inguinal region, especially on making firm and deep pressure, but neither the anterior crural not obturator nerves appear 
to be implicated: there has not been any tendency to maintain the thigh fixed upon the pelvis.

During her illness the menses, though scanty in quantity and attended with pain, have appeared regularly ; and in the intervals there has been slight leucorrhœea.

The bowels are so confined as to render the constant use of aperients necessary; there is slight pain in defæcation, but the evacuations have not been observed to be flattened, or to assume any unusual form. Micturition is painful and difficult, and, when attempted, only a small quantity of urine passes, and much time is consumed before the bladder can be emptied.

But little sleep is obtained without the use of opium. The tongue is clean; the appetite somewhat deficient; the pulse 96, feeble; the sounds of the heart rather harsh. Respiration is natural, and, by external examination, nothing abnormal can be detected in the abdomen. The right lower extremity is wasted, and much smaller than the left.

Per vaginam scrutans.-The pelvis is large, and the vagina capacious; the cavity of the latter is not constricted at any part, nor is there any cicatrix to be discovered. The end of the examining finger impinges on a resisting substance, which appears to be from a quarter to half an inch broad, but neither extremity of it can be reached. It is lying obliquely in the pelvis to the right of the vagina, (the parietes of which can be rolled over it,) and its direction appears to be, stretching from the tuberosity of the right ischium towards the right sacro-iliac-synchondrosis.

The patient's general health was attended to, and improved under the employment of tonics and a generous diet, while the pains were for a time mitigated by belladonna plaster and liniment, but again became excessive on the setting in of the cold weather.

1848.-Jan. 23rd.-At Dr. Lever's request, Mr. Hilton examined the patient to-day, and expressed his conviction that the foreign body was lying obliquely in the pelvis, with its lower extremity firmly fixed near the tuberosity of the 
right ischium, and close to the foramen ovale; and its upper extremity (which seemed a little moveable) extending in the direction of the right sacro-iliac-synchondrosis. It could be distinctly felt through the vagina or rectum, and also by a catheter introduced into the bladder. Its situation was external and to the right of these pelvic viscera. The internal pudic artery was felt pulsating over its lower extremity, but no blood-vessel could be detected over the more central portion of it. Pressure from the vagina upon this part caused an increase of pain in the course of the great ischiatic nerve. The patient was examined in different positions, in order to ascertain how the foreign body might most easily be reached from the vagina. It was felt least distinctly when the patient was lying on her back, and most readily when the patient was placed on her left side; but $\mathbf{M r}$. Hilton thought the operation could more easily be performed with the patient resting on her right side.

Jan. 24th.-She suffered a good deal of pain yesterday after the examination, and had but little sleep last night; and she feels rather low this morning. Pulse 90, rather feeble. Tongue clean and moist. The bowels have been relieved by ol. ricini.

The operation was performed at half-past twelve o'clock. The bladder being first emptied by the catheter, the patient was placed on her right side. Mr. Hilton having felt the foreign body distinctly through the vagina, directed, that in order to force it towards the vagina, and to keep it steady, pressure should be made upon the lower part of the abdomen by an assistant. The parietes of the vagina being separated from each other by the aid of two bone spatulæ, Mr. Hilton placed the end of the forefinger of his left hand upon the extraneous body, and, with a scalpel thus directed, cut down upon it : this was attended with some difficulty in consequence of its edge presenting to his knife. The mesh was laid hold of by a pair of long dressing forceps, and an effort was made to move it, and extract it whole, but without effect, from the fixity of position it had acquired. An aneurism 
needle, armed with a long silk ligature was next introduced through the incision, and placed underneath the mesh, and, after one or two attempts, the double silk was seized with forceps and drawn into the vagina. The loop of silk, being now divided, formed two separate ligatures, which were tied, one round the upper, and the other round the lower, part of the portion of the mesh which had been denuded by the incision. The mesh was then divided between the ligatures with a pair of bone forceps. A long pair of dressing-forceps being now introduced into the vagina, the upper portion of the mesh was seized and withdrawn downwards (measuring four inches in length), and immediately after the lower portion was raised from its position near the foramen ovale, and then brought through the incision. This shorter portion measured one inch and five-eighths in length.

The ligatures werenot used with the view of being of any service in the extraction of the foreign body, beyond keeping the two portions steady after division was effected, and preventing their slipping out of reach.

Not an ounce of blood was lost during the operation, which, although somewhat tedious, was borne by the patient with the greatest fortitude. She merely complained of increased pain and numbness, from having been placed on the affected side. The catheter was passed immediately after the operation, and about $\xi \mathrm{ij}$. of clear, healthy urine withdrawn. Ordered,B. Opii mxxx. st.

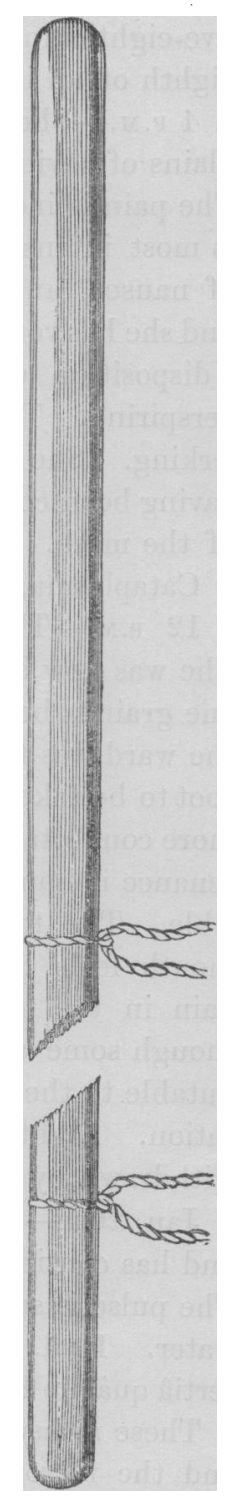


The bone mesh, after removal, measured five inches and five-eighths in length; a quarter of an inch in breadth; onecighth of an inch in thickuess.

4 P.M.-She is lying with her knees drawn up, and complains of having had pain in the abdomen since the operation. The pain is increased by pressure, inspiration, or motion, and is most intense in the right iliac region. There is a feeling of nausea, and of constriction about the scrobiculus cordis, and she has vomited a greenish fluid. She is cold, and has had a disposition to shiver, but the skin is now warmer, and rather perspiring. The countenance is anxious; the pulse 100, and jerking. She has no pain in the right lower extremity, this having been completely relieved immediately after the removal of the mesh.

Cataplasma, lini. abdom. app. Bk. Opii mxxx. st.

12 P.M.-The patient vomited after taking the opium. She was now ordered two grains of solid opium directly, and one grain to be repeated every four hours: the temperature of the ward was to be kept up, and a little beef-tea and arrowroot to be taken occasionally. She has felt much warmer and more comfortable, having less pain and nausea, and the countenance is improved. The pulse 92, full, soft and compressible. The tongue is whitish; but she complains that her mouth feels parched; the skin is rather hot; there is no pain in the head; and the knees arc not drawn up, although some abdominal tenderness remains, probably attributable to the pressure exerted on that part during the operation. Eight ounces of high-coloured acid urine have been withdrawn by the catheter.

Jan. 26th-11 n.s.-The vomiting returned at 1 o'clock, and has continued at intervals, increasing the abdominal pain. The pulse is small and jerking, 110. She was ordered iced water. Hyd. chlorid. gr. j. Pulv. opii gr. fs. in formâ pil. tertiâ quâque horâ; and Catap. lini. abdom. $2^{\text {dis }}$ horis applic.

These measures, together with the syringing of the vagina, and the introduction of the catheter, were persercred in until the crening of the 26th, when, as her gums were 
affected, the pills were discontinued, and a more generous diet was allowed.

From this time, until Feb. 1, the patient continued steadily and progressively to improve; the discharge of pus from the vagina gradually diminishing, and at length ceasing altogether. She rapidly regained her health and strength, and was soon able to walk perfectly well without assistance, not having had the least return of pain or numbness in the right lower extremity since the operation; and on Feb. 22, 1848, she left the hospital perfectly well.

The chief feature in such a case as that which has been related may, by some, be found in the rarity of its occurrence, and in that aspect alone it is interesting : but it certainly leads to more worthy considerations when we reflect on its details; - the pain and distress with which the patient was and would have been afflicted during the detention of the extraneous body in the pelvis, and the risk of danger in the employment of any operative means for its displacement. We have an instance of an extraneous body-a portion of bone nearly six inches long-forced through the walls of the vagina, the pelvic fascia and the cellular tissue, and, in all probability, through the right broad ligament, (or it might have been only pressing this ligament with great tension upwards and backwards,) so as to reach the locality of the lumbo-sacral or first sacral nerve, without causing any important hæmorrhage, or inducing any very serious inflammatory mischief, at that time or subsequently. It is also worthy of notice that, so far as could be ascertained, this piece of bone had not led to any chronic inflammatory deposits in the immediately surrounding surface, nor to any secondary local disturbance, except that produced by its direct pressure upon the nerve. It may be certainly inferred that the upper end of the mesh was not placed upon the anterior crural or obturator nerves, from the whole of the nervous symptoms having been referred to the parts of the body below their peripheral distribution; and on the other hand it seems voL. XXXI. 
to be quite clear that it had rested for some time, and did rest up to the period of the operation, upon some of the nerves forming the sciatic, from the whole of the painful nervous symptoms, the muscular wasting, and diminished temperature having been in accordance with its known muscular and cutaneous associations; and, further, from the pain being increased, by pressure upon the mesh, in the direction of the first sacral foramen, and from the immediate and complete relief obtained by its removal. It would appear that the lower end of the mesh was fixed near the margin of the obturator foramen, to the outer side of the right internal pudic artery. It will be evident also that the mesh could not have perforated from below its then situation through the vagina; indeed, there is every reason to suppose that it had traversed the walls of this canal at a higher point, and had subsequently gravitated to the spot at which it became fixed. It is perhaps worthy of a passing remark, that the long detention of this piece of bone, in contact with living tissues, had not produced any recognizable alteration or change upon any of its surfaces. :

After the operation the patient was severely ill, and it was within a probability that she might have died from inflammation of the pelvic tissues or from extending peritonitis. Knowing well how susceptible and liable to dangerous inflammatory mischief the pelvic fascia and areolar tissue become after surgical operations in the neighbourhood; yet, considering the size of the extraneous body and its remarkable position, the severe pain and atrophy of the limb, together with the increasing deterioration of the general health, which resulted from its presence, and that there did not appear any probability of its being removed by a suppurative or ulcerative process,-we concluded that the operative means employed for its removal were not only justifiable, but to be recommended.*

* The report of the foregoing case was compiled by the Authors from Notes taken by Mr.T. Beavan Rake. 